\title{
RELAXATION OF $p$-GROWTH INTEGRAL FUNCTIONALS UNDER SPACE-DEPENDENT DIFFERENTIAL CONSTRAINTS
}

\author{
ELISA DAVOLI AND IRENE FONSECA
}

Abstract. A representation formula for the relaxation of integral energies

$$
(u, v) \mapsto \int_{\Omega} f(x, u(x), v(x)) d x,
$$

is obtained, where $f$ satisfies $p$-growth assumptions, $1<p<+\infty$, and the fields $v$ are subjected to space-dependent first order linear differential constraints in the framework of $\mathscr{A}$-quasiconvexity with variable coefficients.

\section{INTRODUCTION}

The analysis of constrained relaxation problems is a central question in materials science. Many applications in continuum mechanics and, in particular, in magnetoelasticity, rely on the characterization of minimizers of non-convex multiple integrals of the type

or

$$
u \mapsto \int_{\Omega} f\left(x, u(x), \nabla u(x), \ldots, \nabla^{k} u(x)\right) d x
$$

$$
(u, v) \mapsto \int_{\Omega} f(x, u(x), v(x)) d x,
$$

where $\Omega$ is an open, bounded subset of $\mathbb{R}^{N}, u: \Omega \rightarrow \mathbb{R}^{m}, m \in \mathbb{N}$, and the fields $v: \Omega \rightarrow \mathbb{R}^{d}, d \in \mathbb{N}$, satisfy partial differential constraints of the type " $\mathscr{A} v=0$ " other than $\operatorname{curl} v=0$ (see e.g. [5, 9]).

In this paper we provide a representation formula for the relaxation of non-convex integral energies of the form (1.1), in the case in which the energy density $f$ satisfies $p$-growth assumptions, and the fields $v$ are subjected to linear first-order space-dependent differential constraints.

The natural framework to study this family of relaxation problems is within the theory of $\mathscr{A}$ quasiconvexity with variable coefficients. In order to present this notion, we need to introduce some notation.

For $i=1 \cdots, N$, let $A^{i} \in C^{\infty}\left(\mathbb{R}^{N} ; \mathbb{M}^{l \times d}\right) \cap W^{1, \infty}\left(\mathbb{R}^{N} ; \mathbb{M}^{l \times d}\right)$, let $1<p<+\infty$, and consider the differential operator

defined as

$$
\mathscr{A}: L^{p}\left(\Omega ; \mathbb{R}^{d}\right) \rightarrow W^{-1, p}\left(\Omega ; \mathbb{R}^{l}\right), \quad d, l \in \mathbb{N},
$$

$$
\mathscr{A} v:=\sum_{i=1}^{N} A^{i}(x) \frac{\partial v(x)}{\partial x_{i}}
$$

for every $v \in L^{p}\left(\Omega ; \mathbb{R}^{d}\right)$, where (1.2) is to be interpreted in the sense of distributions. Assume that the symbol $\mathbb{A}: \mathbb{R}^{N} \times \mathbb{R}^{N} \rightarrow \mathbb{M}^{l \times d}$,

$$
\mathbb{A}(x, w):=\sum_{i=1}^{N} A^{i}(x) w_{i} \quad \text { for }(x, w) \in \mathbb{R}^{N} \times \mathbb{R}^{N},
$$

2010 Mathematics Subject Classification. 49J45; 35D99; 49K20.

Key words and phrases. Relaxation, $\mathscr{A}$-quasiconvexity. 
satisfies the uniform constant rank condition (see [22])

$$
\operatorname{rank} \mathbb{A}(x, w)=r \quad \text { for every } x \in \mathbb{R}^{N} \text { and } w \in \mathbb{S}^{n-1} \text {. }
$$

Let $Q$ be the unit cube in $\mathbb{R}^{N}$ with sides parallel to the coordinate axis, i.e.,

$$
Q:=\left(-\frac{1}{2}, \frac{1}{2}\right)
$$

Denote by $C_{\text {per }}^{\infty}\left(\mathbb{R}^{N} ; \mathbb{R}^{m}\right)$ the set of $\mathbb{R}^{m}$-valued smooth maps that are $Q$-periodic in $\mathbb{R}^{N}$, and for every $x \in \Omega$ consider the set

$$
\mathcal{C}_{x}:=\left\{w \in C_{\text {per }}^{\infty}\left(\mathbb{R}^{N} ; \mathbb{R}^{m}\right): \int_{Q} w(y) d y=0, \text { and } \sum_{i=1}^{N} A^{i}(x) \frac{\partial w(y)}{\partial y_{i}}=0\right\} .
$$

Let $f: \Omega \times \mathbb{R}^{m} \times \mathbb{R}^{d} \rightarrow[0,+\infty)$ be a Carathéodory function. The $\mathscr{A}$-quasiconvex envelope of $f(x, u, \cdot)$ for $x \in \Omega$ and $u \in \mathbb{R}^{m}$ is defined for $\xi \in \mathbb{R}^{d}$ as

$$
Q_{\mathscr{A}(x)} f(x, u, \xi):=\inf \left\{\int_{Q} f(x, u, \xi+w(y)) d y: w \in \mathcal{C}_{x}\right\} .
$$

We say that $f$ is $\mathscr{A}$-quasiconvex if $f(x, u, \xi)=Q_{\mathscr{A}(x)} f(x, u, \xi)$ for a.e. $x \in \Omega$, and for all $u \in \mathbb{R}^{m}$ and $\xi \in \mathbb{R}^{d}$.

The notion of $\mathscr{A}$-quasiconvexity was first introduced by B. Dacorogna in [8], and extensively characterized in [17] by I. Fonseca and S. Müller for operators $\mathscr{A}$ defined as in (1.2), satisfying the constant rank condition (1.3), and having constant coefficients,

$$
A^{i}(x) \equiv A^{i} \in \mathbb{M}^{l \times d} \quad \text { for every } x \in \mathbb{R}^{N}, i=1, \ldots, N .
$$

In that paper the authors proved (see [17, Theorems 3.6 and 3.7 ]) that under $p$-growth assumptions on the energy density $f, \mathscr{A}$-quasiconvexity is necessary and sufficient for the lower-semicontinuity of integral functionals

$$
I(u, v):=\int_{\Omega} f(x, u(x), v(x)) d x \quad \text { for every }(u, v) \in L^{p}\left(\Omega ; \mathbb{R}^{m}\right) \times L^{p}\left(\Omega ; \mathbb{R}^{d}\right)
$$

along sequences $\left(u^{n}, v^{n}\right)$ satisfying $u^{n} \rightarrow u$ in measure, $v^{n} \rightarrow v$ in $L^{p}\left(\Omega ; \mathbb{R}^{d}\right)$, and $\mathscr{A} v^{n} \rightarrow 0$ in $W^{-1, p}(\Omega)$. We remark that in the framework $\mathscr{A}=$ curl, i.e., when $v^{n}=\nabla \phi^{n}$ for some $\phi^{n} \in$ $W^{1, p}\left(\Omega ; \mathbb{R}^{m}\right), d=n \times m, \mathscr{A}$-quasiconvexity reduces to Morrey's notion of quasiconvexity.

The analysis of properties of $\mathscr{A}$-quasiconvexity for operators with constant coefficients was extended in the subsequent paper [6], where A. Braides, I. Fonseca and G. Leoni provided an integral representation formula for relaxation problems under $p$-growth assumptions on the energy density, and presented (via $\Gamma$-convergence) homogenization results for periodic integrands evaluated along $\mathscr{A}$-free fields. These homogenization results were later generalized in [13], where I. Fonseca and S. Krömer worked under weaker assumptions on the energy density $f$. In $[19,20]$, simultaneous homogenization and dimension reduction was studied in the framework of $\mathscr{A}$-quasiconvexity with constant coefficients. Oscillations and concentrations generated by $\mathscr{A}$-free mappings are the subject of [14]. Very recently an analysis of the case in which the energy density is nonpositive has been carried out in [18], and applications to the theory of compressible Euler systems have been studied in [7]. A parallel analysis for operators with constant coefficients and under linear growth assumptions for the energy density has been developed in $[1,4,15,21]$. A very general characterization in this setting has been obtained in [2], following the new insight in [12].

The theory of $\mathscr{A}$-quasiconvexity for operators with variable coefficients has been characterized by P. Santos in [23]. Homogenization results in this setting have been obtained in [10] and [11]. 
This paper is devoted to proving a representation result for the relaxation of integral energies in the framework of $\mathscr{A}$-quasiconvexity with variable coefficients. To be precise, let $1<p, q<+\infty$, $d, m, l \in \mathbb{N}$, and consider a Carathéodory function $f: \Omega \times \mathbb{R}^{m} \times \mathbb{R}^{d} \rightarrow[0,+\infty)$ satisfying

$$
\text { (H) } \quad 0 \leq f(x, u, v) \leq C\left(1+|u|^{p}+|v|^{q}\right), \quad 1<p, q<+\infty,
$$

for a.e. $x \in \Omega$, and all $(u, v) \in \mathbb{R}^{m} \times \mathbb{R}^{d}$, with $C>0$.

Denoting by $\mathcal{O}(\Omega)$ the collection of open subsets of $\Omega$, for every $D \in \mathcal{O}(\Omega), u \in L^{p}\left(\Omega ; \mathbb{R}^{m}\right)$ and $v \in L^{q}\left(\Omega ; \mathbb{R}^{d}\right)$ with $\mathscr{A} v=0$, we define

$$
\begin{aligned}
\mathcal{I}((u, v), D):=\inf \{ & \liminf _{n \rightarrow+\infty} \int_{D} f\left(x, u_{n}(x), v_{n}(x)\right): u_{n} \rightarrow u \quad \text { strongly in } L^{p}\left(\Omega ; \mathbb{R}^{m}\right), \\
& \left.v_{n} \rightarrow v \quad \text { weakly in } L^{q}\left(\Omega ; \mathbb{R}^{d}\right) \text { and } \mathscr{A} v_{n} \rightarrow 0 \quad \text { strongly in } W^{-1, q}\left(\Omega ; \mathbb{R}^{l}\right)\right\} .
\end{aligned}
$$

Our main result is the following.

Theorem 1.1. Let $\mathscr{A}$ be a first order differential operator with variable coefficients, satisfying (1.3). Let $f: \Omega \times \mathbb{R}^{m} \times \mathbb{R}^{d} \rightarrow[0,+\infty)$ be a Carathéodory function satisfying $(\mathrm{H})$. Then,

$$
\int_{D} Q_{\mathscr{A}(x)} f(x, u(x), v(x)) d x=\mathcal{I}((u, v), D)
$$

for all $D \in \mathcal{O}(\Omega), u \in L^{p}\left(\Omega ; \mathbb{R}^{m}\right)$ and $v \in L^{q}\left(\Omega ; \mathbb{R}^{d}\right)$ with $\mathscr{A} v=0$.

Adopting the "blow-up" method introduced in [16], the proof of the theorem consists in showing that the functional $\mathcal{I}((u, v), \cdot)$ is the trace of a Radon measure absolutely continuous with respect to the restriction of the Lebesgue measure $\mathcal{L}^{N}$ to $\Omega$, and proving that for a.e. $x \in \Omega$ the Radon-Nicodym derivative $\frac{d \mathcal{I}((u, v) \cdot)(x)}{d \mathcal{L}^{N}}$ coincides with the $\mathscr{A}$-quasiconvex envelope of $f$.

The arguments used are a combination of the ideas from [6, Theorem 1.1] and from [23]. The main difference with [6, Theorem 1.1], which reduces to our setting in the case in which the operator $\mathscr{A}$ has constant coefficients, is in the fact that while defining the operator $\mathcal{I}$ in (1.4) we can not work with exact solutions of the PDE, but instead we need to study sequences of asymptotically $\mathscr{A}$-vanishing fields. As pointed out in [23], in the case of variable coefficients the natural framework is the context of pseudo-differential operators. In this setting, we don't know how to project directly onto the kernel of the differential constraint, but we are able to construct an "approximate" projection operator $P$ such that for every field $v \in L^{p}$, the $W^{-1, p}$ norm of $\mathscr{A} P v$ is controlled by the $W^{-1, p}$ norm of $v$ itself (we refer to [23, Subsection 2.1] for a detailed explanation of this issue and to the references therein for a treatment of the main properties of pseudo-differential operators). For the same reason, in the proof of the inequality

$$
\frac{d \mathcal{I}((u, v) \cdot)(x)}{d \mathcal{L}^{N}} \leq Q_{\mathscr{A}(x)} f(x, u(x), v(x)) \quad \text { for a.e. } x \in \Omega,
$$

an equi-integrability argument is needed (see Proposition 3.2). We also point out that the representation formula in Theorem 1.1 was obtained in a simplified setting in [11] as a corollary of the main homogenization result. Here we provide an alternative, direct proof, which does not rely on homogenization techniques.

The paper is organized as follows: in Section 2 we establish the main assumptions on the differential operator $\mathscr{A}$ and we recall some preliminary results on $\mathscr{A}$-quasiconvexity with variable coefficients. Section 3 is devoted to the proof of Theorem 1.1.

\section{Notation}

Throughout the paper $\Omega \subset \mathbb{R}^{N}$ is a bounded open set, $1<p, q<+\infty, \mathcal{O}(\Omega)$ is the set of open subsets of $\Omega, Q$ denotes the unit cube in $\mathbb{R}^{N}, Q\left(x_{0}, r\right)$ and $B\left(x_{0}, r\right)$ are, respectively, the open cube and the 
open ball in $\mathbb{R}^{N}$, with center $x_{0}$ and radius $r$. Given an exponent $1<q<+\infty$, we denote by $q^{\prime}$ its conjugate exponent, i.e., $q^{\prime} \in(1,+\infty)$ is such that

$$
\frac{1}{q}+\frac{1}{q^{\prime}}=1
$$

Whenever a map $v \in L^{q}, C^{\infty}, \cdots$ is $Q$-periodic, that is

$$
v\left(x+e_{i}\right)=v(x) \quad i=1, \cdots, N,
$$

for a.e. $x \in \mathbb{R}^{N},\left\{e_{1}, \cdots, e_{N}\right\}$ being the standard basis of $\mathbb{R}^{N}$, we write $v \in L_{\text {per }}^{q}, C_{\text {per }}^{\infty}, \ldots$ We implicitly identify the spaces $L^{q}(Q)$ and $L_{\text {per }}^{q}\left(\mathbb{R}^{N}\right)$.

We adopt the convention that $C$ will denote a generic constant, whose value may change from line to line in the same formula.

\section{Preliminary Results}

In this section we introduce the main assumptions on the differential operator $\mathscr{A}$ and we recall some preliminary results about $\mathscr{A}$-quasiconvexity.

For $i=1, \cdots, N, x \in \mathbb{R}^{N}$, consider the linear operators $A^{i}(x) \in \mathbb{M}^{l \times d}$, with $A^{i} \in C^{\infty}\left(\mathbb{R}^{N} ; \mathbb{M}^{l \times d}\right) \cap$ $W^{1, \infty}\left(\mathbb{R}^{N} ; \mathbb{M}^{l \times d}\right)$. For every $v \in L^{q}\left(\Omega ; \mathbb{R}^{d}\right)$ we set

$$
\mathscr{A} v:=\sum_{i=1}^{N} A^{i}(x) \frac{\partial v(x)}{\partial x_{i}} \in W^{-1, q}\left(\Omega ; \mathbb{R}^{l}\right) .
$$

The symbol $\mathbb{A}: \mathbb{R}^{N} \times \mathbb{R}^{N} \backslash\{0\} \rightarrow \mathbb{M}^{l \times d}$ associated to the differential operator $\mathscr{A}$ is

$$
\mathbb{A}(x, \lambda):=\sum_{i=1}^{N} A^{i}(x) \lambda_{i} \in \mathbb{M}^{l \times d}
$$

for every $x \in \mathbb{R}^{N}, \lambda \in \mathbb{R}^{N} \backslash\{0\}$. We assume that $\mathscr{A}$ satisfies the following uniform constant rank condition:

$$
\operatorname{rank}\left(\sum_{i=1}^{N} A^{i}(x) \lambda_{i}\right)=r \quad \text { for all } x \in \mathbb{R}^{N} \text { and } \lambda \in \mathbb{R}^{N} \backslash\{0\} .
$$

For every $x \in \mathbb{R}^{N}, \lambda \in \mathbb{R}^{N} \backslash\{0\}$, let $\mathbb{P}(x, \lambda): \mathbb{R}^{d} \rightarrow \mathbb{R}^{d}$ be the linear projection on $\operatorname{Ker} \mathbb{A}(x, \lambda)$, and let $\mathbb{Q}(x, \lambda): \mathbb{R}^{l} \rightarrow \mathbb{R}^{d}$ be the linear operator given by

$$
\begin{aligned}
& \mathbb{Q}(x, \lambda) \mathbb{A}(x, \lambda) v:=v-\mathbb{P}(x, \lambda) v \text { for all } v \in \mathbb{R}^{d}, \\
& \mathbb{Q}(x, \lambda) \xi=0 \text { if } \xi \notin \text { Range } \mathbb{A}(x, \lambda) .
\end{aligned}
$$

The main properties of $\mathbb{P}(\cdot, \cdot)$ and $\mathbb{Q}(\cdot, \cdot)$ are recalled in the following proposition (see e.g. $[23$, Subsection 2.1]).

Proposition 2.1. Under the constant rank condition (2.1), for every $x \in \mathbb{R}^{N}$ the operators $\mathbb{P}(x, \cdot)$ and $\mathbb{Q}(x, \cdot)$ are, respectively, 0 -homogeneous and $(-1)$-homogeneous. In addition, $\mathbb{P} \in C^{\infty}\left(\mathbb{R}^{N} \times \mathbb{R}^{N} \backslash\right.$ $\left.\{0\} ; \mathbb{M}^{d \times d}\right)$ and $\mathbb{Q} \in C^{\infty}\left(\mathbb{R}^{N} \times \mathbb{R}^{N} \backslash\{0\} ; \mathbb{M}^{d \times l}\right)$.

Let $\eta \in C_{c}^{\infty}(\Omega ;[0,1]), \eta=1$ in $\Omega^{\prime}$ for some $\Omega^{\prime} \subset \subset \Omega$. We denote by $\mathbb{A}_{\eta}$ the symbol

$$
\mathbb{A}_{\eta}(x, \lambda):=\sum_{i=1}^{N} \eta(x) A^{i}(x) \lambda_{i},
$$

for every $x \in \mathbb{R}^{N}, \lambda \in \mathbb{R}^{N} \backslash\{0\}$, and by $\mathscr{A}_{\eta}$ the corresponding pseudo-differential operator (see [23, Subsection 2.1] for an overview of the main properties of pseudo-differential operators). Let $\chi \in C^{\infty}\left(\mathbb{R}^{+} ; \mathbb{R}\right)$ be such that $\chi(|\lambda|)=0$ for $|\lambda|<1$ and $\chi(|\lambda|)=1$ for $|\lambda|>2$. Let also $P_{\eta}$ be the operator associated to the symbol

$$
\mathbb{P}_{\eta}(x, \lambda):=\eta^{2}(x) \mathbb{P}(x, \lambda) \chi(|\lambda|)
$$


for every $x \in \mathbb{R}^{N}, \lambda \in \mathbb{R}^{N} \backslash\{0\}$. The following proposition (see [23, Theorem 2.2 and Subsection 2.1]) collects the main properties of the operators $P_{\eta}$ and $\mathscr{A}_{\eta}$.

Proposition 2.2. Let $1<q<+\infty$, and let $\mathscr{A}_{\eta}$ and $P_{\eta}$ be the pseudo-differential operators associated with the symbols (2.2) and (2.3), respectively. Then there exists a constant $C$ such that

$$
\left\|P_{\eta} v\right\|_{L^{q}\left(\Omega ; \mathbb{R}^{d}\right)} \leq C\|v\|_{L^{q}\left(\Omega ; \mathbb{R}^{d}\right)}
$$

for every $v \in L^{q}\left(\Omega ; \mathbb{R}^{d}\right)$, and

$$
\begin{aligned}
& \left\|P_{\eta} v\right\|_{W^{-1, q}\left(\Omega ; \mathbb{R}^{d}\right)} \leq C\|v\|_{W^{-1, q}\left(\Omega ; \mathbb{R}^{d}\right)}, \\
& \left\|v-P_{\eta} v\right\|_{L^{q}\left(\Omega ; \mathbb{R}^{d}\right)} \leq C\left(\left\|\mathscr{A}_{\eta} v\right\|_{W^{-1, q}\left(\Omega ; \mathbb{R}^{l}\right)}+\|v\|_{W^{-1, q}\left(\Omega ; \mathbb{R}^{d}\right)}\right), \\
& \left\|\mathscr{A}_{\eta} P_{\eta} v\right\|_{W^{-1, q}\left(\Omega ; \mathbb{R}^{l}\right)} \leq C\|v\|_{W^{-1, q}\left(\Omega ; \mathbb{R}^{d}\right)}
\end{aligned}
$$

for every $v \in W^{-1, q}\left(\Omega ; \mathbb{R}^{d}\right)$.

\section{Proof of Theorem 1.1}

Before proving Theorem 1.1 we state and prove a decomposition lemma, which generalizes [17, Lemma 2.15] to the case of operators with variable coefficients.

Lemma 3.1. Let $1<q<+\infty$. Let $\mathscr{A}$ be a first order differential operator with variable coefficients, satisfying (2.1). Let $v \in L^{q}\left(\Omega ; \mathbb{R}^{d}\right)$, and let $\left\{v_{n}\right\}$ be a bounded sequence in $L^{q}\left(\Omega ; \mathbb{R}^{d}\right)$ such that

$$
\begin{aligned}
& v_{n} \rightarrow v \quad \text { weakly in } L^{q}\left(\Omega ; \mathbb{R}^{d}\right), \\
& \mathscr{A} v_{n} \rightarrow 0 \text { strongly in } W^{-1, q}\left(\Omega ; \mathbb{R}^{l}\right), \\
& \left\{v_{n}\right\} \text { generates the Young measure } \nu .
\end{aligned}
$$

Then, there exists a q-equiintegrable sequence $\left\{\tilde{v}_{n}\right\} \subset L^{q}\left(\Omega ; \mathbb{R}^{d}\right)$ such that

$$
\begin{aligned}
& \mathscr{A} \tilde{v}_{n} \rightarrow 0 \quad \text { strongly in } W^{-1, s}\left(\Omega ; \mathbb{R}^{l}\right) \quad \text { for every } 1<s<q, \\
& \int_{\Omega} \tilde{v}_{n}(x) d x=\int_{\Omega} v(x) d x, \\
& \tilde{v}_{n}-v_{n} \rightarrow 0 \text { strongly in } L^{s}\left(\Omega ; \mathbb{R}^{d}\right) \quad \text { for every } 1<s<q, \\
& \tilde{v}_{n} \rightarrow v \text { weakly in } L^{q}\left(\Omega ; \mathbb{R}^{d}\right) .
\end{aligned}
$$

In addition, if $\Omega \subset Q$ then we can construct the sequence $\left\{\tilde{v}^{n}\right\}$ so that $\tilde{v}_{n}-v \in L_{\text {per }}^{q}\left(\mathbb{R}^{N} ; \mathbb{R}^{d}\right)$ for every $n \in \mathbb{N}$.

Proof. Arguing as in the first part of [23, Proof of Theorem 1.1], we construct a q-equiintegrable sequence $\left\{\hat{v}_{n}\right\}$ satisfying (3.1), (3.2) and (3.3). The conclusion follows by setting $\tilde{v}_{n}:=\hat{v}_{n}-\int_{\Omega} \hat{v}_{n}(x) d x+$ $\int_{\Omega} v(x) d x$.

In the case in which $\Omega \subset Q$, let $\left\{\varphi^{i}\right\}$ be a sequence of cut-off functions in $Q$ with $0 \leq \varphi^{i} \leq 1$ in $Q$, such that $\varphi^{i}=0$ on $Q \backslash \Omega$ and $\varphi^{i} \rightarrow 1$ pointwise in $\Omega$. Define $w_{n}^{i}:=\varphi^{i}\left(\hat{v}_{n}-v\right)$. By (3.3) for every $\psi \in L^{q^{\prime}}\left(\Omega ; \mathbb{R}^{d}\right)$ we have

$$
\lim _{i \rightarrow+\infty} \lim _{n \rightarrow+\infty} \int_{\Omega} w_{n}^{i}(x) \psi(x) d x=0 .
$$

By (3.1), (3.2), and the compact embedding of $L^{q}\left(\Omega ; \mathbb{R}^{d}\right)$ into $W^{-1, q}\left(\Omega ; \mathbb{R}^{d}\right)$, there holds

$$
\mathscr{A} w_{n}^{i}=\varphi^{i} \mathscr{A} \hat{v}_{n}+\left(\sum_{j=1}^{N} A^{j} \frac{\partial \varphi^{i}}{\partial x_{j}}\right) \hat{v}_{n} \rightarrow 0 \quad \text { strongly in } W^{-1, s}\left(\Omega ; \mathbb{R}^{l}\right)
$$

as $n \rightarrow+\infty$, for every $1<s<q$. Extending the maps $w_{n}^{i}$ outside $Q$ by periodicity, by the metrizability of the weak topology on bounded sets and by Attouch's diagonalization lemma (see [3, Lemma 1.15 and Corollary 1.16]), we obtain a sequence

$$
w_{n}:=w_{n}^{i(n)}
$$


with $\left\{w_{n}\right\} \subset L_{\text {per }}^{q}\left(\mathbb{R}^{N} ; \mathbb{R}^{d}\right)$, and such that $w_{n}+v$ satisfies (3.1), (3.2) and (3.3). The thesis follows by setting

$$
\tilde{v}_{n}:=w_{n}-\int_{\Omega} w_{n}(x) d x+v
$$

The following proposition will allow us to neglect vanishing perturbations of $q$-equiintegrable sequences.

Proposition 3.2. For every $n \in \mathbb{N}$, let $f_{n}: Q \times \mathbb{R}^{d} \rightarrow[0,+\infty)$ be a continuous function. Assume that there exists a constant $C>0$ such that, for $q>1$,

$$
\sup _{n \in \mathbb{N}} f_{n}(y, \xi) \leq C\left(1+|\xi|^{q}\right) \quad \text { for every } y \in Q \text { and } \xi \in \mathbb{R}^{d},
$$

and that the sequence $\left\{f_{n}(y, \cdot)\right\}$ is equicontinuous in $\mathbb{R}^{d}$, uniformly in $y$. Let $\left\{w_{n}\right\}$ be a q-equiintegrable sequence in $L^{q}\left(Q ; \mathbb{R}^{d}\right)$, and let $\left\{v_{n}\right\} \subset L^{q}\left(Q ; \mathbb{R}^{d}\right)$ be such that

$$
v_{n} \rightarrow 0 \text { strongly in } L^{q}\left(Q ; \mathbb{R}^{d}\right) \text {. }
$$

Then

$$
\lim _{n \rightarrow+\infty}\left|\int_{Q} f_{n}\left(y, w_{n}(y)\right) d y-\int_{Q} f_{n}\left(y, v_{n}(y)+w_{n}(y)\right) d y\right|=0 .
$$

Proof. Fix $\eta>0$. In view of (3.5), the sequence $\left\{C\left(1+\left|v_{n}\right|^{q}+\left|w_{n}\right|^{q}\right)\right\}$ is equiintegrable in $Q$, thus there exists $0<\varepsilon<\frac{\eta}{3}$ such that

$$
\sup _{n \in \mathbb{N}} \int_{A} C\left(1+\left|v_{n}(y)\right|^{q}+\left|w_{n}(y)\right|^{q}\right) d y<\frac{\eta}{3}
$$

for every $A \subset Q$ with $|A|<\varepsilon$. By the $q$-equiintegrability of $\left\{w_{n}\right\}$ and $\left\{v_{n}\right\}$, and by Chebyshev's inequality there holds

$$
\left|Q \cap\left(\left\{\left|w_{n}\right|>M\right\} \cup\left\{\left|v_{n}\right|>M\right\}\right)\right| \leq \frac{1}{M^{q}} \int_{Q}\left(\left|w_{n}(y)\right|^{q}+\left|v_{n}(y)\right|^{q}\right) d y \leq \frac{C}{M^{q}}
$$

for every $n \in \mathbb{N}$. Therefore, there exists $M_{0}$ satisfying

$$
\sup _{n \in \mathbb{N}}\left|Q \cap\left(\left\{\left|w_{n}\right|>M_{0}\right\} \cup\left\{\left|v_{n}\right|>M_{0}\right\}\right)\right| \leq \frac{\varepsilon}{2} .
$$

By the uniform equicontinuity of the sequence $\left\{f_{n}(y, \cdot)\right\}$, there exists $\delta>0$ such that, for every $\xi_{1}, \xi_{2} \in \overline{B\left(0, M_{0}\right)}$, with $\left|\xi_{1}-\xi_{2}\right|<\delta$, we have

$$
\sup _{y \in Q}\left|f_{n}\left(y, \xi_{1}\right)-f_{n}\left(y, \xi_{2}\right)\right|<\varepsilon
$$

for every $n \in \mathbb{N}$. By (3.5) and Egoroff's theorem, there exists a set $E_{\varepsilon} \subset Q,\left|E_{\varepsilon}\right|<\frac{\varepsilon}{2}$, such that

$$
v_{n} \rightarrow 0 \text { uniformly in } Q \backslash E_{\varepsilon},
$$

and, in particular,

$$
\left|v_{n}(x)\right|<\delta \text { for a.e. } x \in Q \backslash E_{\varepsilon},
$$

for every $n \geq n_{0}$, for some $n_{0} \in \mathbb{N}$.

We observe that

$$
\begin{aligned}
\int_{Q} f_{n}\left(y, v_{n}(y)+w_{n}(y)\right) d y= & \int_{Q \cap\left\{\left|w_{n}\right| \leq M_{0}\right\} \cap\left\{\left|v_{n}\right| \leq M_{0}\right\}} f_{n}\left(y, v_{n}(y)+w_{n}(y)\right) d y \\
& +\int_{Q \cap\left(\left\{\left|w_{n}\right|>M_{0}\right\} \cup\left\{\left|v_{n}\right|>M_{0}\right\}\right)} f_{n}\left(y, v_{n}(y)+w_{n}(y)\right) d y .
\end{aligned}
$$


The first term in the right-hand side of (3.10) can be further decomposed as

$$
\begin{aligned}
\int_{Q \cap\left\{\left|w_{n}\right| \leq M_{0}\right\} \cap\left\{\left|v_{n}\right| \leq M_{0}\right\}} f_{n}\left(y, v_{n}(y)+w_{n}(y)\right) d y \\
=\int_{\left(Q \backslash E_{\varepsilon}\right) \cap\left\{\left|w_{n}\right| \leq M_{0}\right\} \cap\left\{\left|v_{n}\right| \leq M_{0}\right\}} f_{n}\left(y, v_{n}(y)+w_{n}(y)\right) d y \\
\quad+\int_{E_{\varepsilon} \cap\left\{\left|w_{n}\right| \leq M_{0}\right\} \cap\left\{\left|v_{n}\right| \leq M_{0}\right\}} f_{n}\left(y, v_{n}(y)+w_{n}(y)\right) d y \\
=\int_{\left(Q \backslash E_{\varepsilon}\right) \cap\left\{\left|w_{n}\right| \leq M_{0}\right\} \cap\left\{\left|v_{n}\right| \leq M_{0}\right\}} f_{n}\left(y, w_{n}(y)\right) d y \\
\quad+\int_{\left(Q \backslash E_{\varepsilon}\right) \cap\left\{\left|w_{n}\right| \leq M_{0}\right\} \cap\left\{\left|v_{n}\right| \leq M_{0}\right\}}\left(f_{n}\left(y, v_{n}(y)+w_{n}(y)\right)-f_{n}\left(y, w_{n}(y)\right)\right) d y \\
\quad+\int_{E_{\varepsilon} \cap\left\{\left|w_{n}\right| \leq M_{0}\right\} \cap\left\{\left|v_{n}\right| \leq M_{0}\right\}} f_{n}\left(y, v_{n}(y)+w_{n}(y)\right) d y \\
=\int_{Q} f_{n}\left(y, w_{n}(y)\right) d y-\int_{E_{\varepsilon} \cap\left\{\left|w_{n}\right| \leq M_{0}\right\} \cap\left\{\left|v_{n}\right| \leq M_{0}\right\}} f_{n}\left(y, w_{n}(y)\right) d y \\
\quad-\int_{Q \cap\left(\left\{\left|w_{n}\right|>M_{0}\right\} \cup\left\{\left|v_{n}\right|>M_{0}\right\}\right)} f_{n}\left(y, w_{n}(y)\right) d y \\
\quad+\int_{\left(Q \backslash E_{\varepsilon}\right) \cap\left\{\left|w_{n}\right| \leq M_{0}\right\} \cap\left\{\left|v_{n}\right| \leq M_{0}\right\}}\left(f_{n}\left(y, v_{n}(y)+w_{n}(y)\right)-f_{n}\left(y, w_{n}(y)\right)\right) d y \\
\quad+\int_{E_{\varepsilon} \cap\left\{\left|w_{n}\right| \leq M_{0}\right\} \cap\left\{\left|v_{n}\right| \leq M_{0}\right\}} f_{n}\left(y, v_{n}(y)+w_{n}(y)\right) d y .
\end{aligned}
$$

We observe that by (3.7)

$$
\left|E_{\varepsilon} \cup\left(\left\{\left|w_{n}\right|>M_{0}\right\} \cup\left\{\left|v_{n}\right|>M_{0}\right\}\right)\right|<\varepsilon .
$$

Hence, for $n \geq n_{0}$, by (3.4), (3.6), (3.8), and (3.9) we deduce the estimate

$$
\begin{aligned}
& \left|\int_{Q} f_{n}\left(y, w_{n}(y)\right) d y-\int_{Q} f_{n}\left(y, v_{n}(y)+w_{n}(y)\right) d y\right| \\
& \quad \leq \varepsilon+\int_{E_{\varepsilon} \cup\left(\left\{\left|w_{n}\right|>M_{0}\right\} \cup\left\{\left|v_{n}\right|>M_{0}\right\}\right)} 2 C\left(1+\left|w_{n}(y)\right|^{p}+\left|v_{n}(y)\right|^{p}\right) d y \leq \varepsilon+\frac{2 \eta}{3} .
\end{aligned}
$$

The thesis follows by the arbitrariness of $\eta$.

We now prove our main result.

Proof of Theorem 1.1. The proof is subdivided into 4 steps. Steps 1 and 2 follow along the lines of [6, Proof of Theorem 1.1]. Step 3 is obtained by modifying [6, Lemma 3.5], whereas Step 4 follows by adapting an argument in [23, Proof of Theorem 1.2]. We only outline the main ideas of Steps 1 and 2 for convenience of the reader, whilst we provide more details for Steps 3 and 4 .

Step 1:

The first step consists in showing that

$$
\begin{gathered}
\mathcal{I}((u, v), D)=\inf \left\{\liminf _{n \rightarrow+\infty} \int_{D} f\left(x, u(x), v_{n}(x)\right) d x:\left\{v_{n}\right\} \text { is } q \text { - equiintegrable },\right. \\
\mathscr{A} v_{n} \rightarrow 0 \text { strongly in } W^{-1, s}\left(D ; \mathbb{R}^{l}\right) \text { for every } 1<s<q \\
\text { and } \left.v_{n} \rightarrow v \text { weakly in } L^{q}\left(D ; \mathbb{R}^{d}\right)\right\} .
\end{gathered}
$$

This identification is proved by adapting [6, Proof of Lemma 3.1]. The only difference is the application of Lemma 3.1 instead of [6, Proposition 2.3 (i)].

Step 2:

The second step is the proof that $\mathcal{I}((u, v), \cdot)$ is the trace of a Radon measure absolutely continuous 
with respect to $\mathcal{L}^{N}\lfloor\Omega$. This follows as a straightforward adaptation of [6, Lemma 3.4]. The only modifications are due to the fact that [6, Proposition 2.3 (i)] and [6, Lemma 3.1] are now replaced by Lemma 3.1 and Step 1.

Step 3:

We claim that

$$
\frac{d \mathcal{I}((u, v), \cdot)}{d \mathcal{L}^{N}}\left(x_{0}\right) \geq Q_{\mathscr{A}\left(x_{0}\right)} f\left(x_{0}, u\left(x_{0}\right), v\left(x_{0}\right)\right) \quad \text { for a.e. } x_{0} \in \Omega .
$$

Indeed, since $g(x, \xi):=f(x, u(x), \xi)$ is a Carathéodory function, by Scorza-Dragoni Theorem there exists a sequence of compact sets $K_{j} \subset \Omega$ such that

$$
\left|\Omega \backslash K_{j}\right| \leq \frac{1}{j}
$$

and the restriction of $g$ to $K_{j} \times \mathbb{R}^{d}$ is continuous. Hence, the set

$$
\omega:=\bigcup_{j=1}^{+\infty}\left(K_{j} \cap K_{j}^{*}\right) \cap \mathcal{L}(u, v),
$$

where $K_{j}^{*}$ is the set of Lebesgue point for the characteristic function of $K_{j}$ and $\mathcal{L}(u, v)$ is the set of Lebesgue points of $u$ and $v$, is such that

$$
|\Omega \backslash \omega| \leq\left|\Omega \backslash K_{j}\right| \leq \frac{1}{j} \quad \text { for every } j,
$$

and so $|\Omega \backslash \omega|=0$. Let $x_{0} \in \omega$ be such that

$$
\lim _{r \rightarrow 0^{+}} \frac{1}{r^{N}} \int_{Q\left(x_{0}, r\right)}\left|u(x)-u\left(x_{0}\right)\right|^{p} d x=\lim _{r \rightarrow 0^{+}} \frac{1}{r^{N}} \int_{Q\left(x_{0}, r\right)}\left|v(x)-v\left(x_{0}\right)\right|^{q} d x=0,
$$

and

$$
\frac{d \mathcal{I}((u, v), \cdot)}{d \mathcal{L}^{N}}\left(x_{0}\right)=\lim _{r \rightarrow 0^{+}} \frac{\mathcal{I}\left((u, v), Q\left(x_{0}, r\right)\right)}{r^{N}}<+\infty,
$$

where the sequence of radii $r$ is such that $\mathcal{I}\left((u, v), \partial Q\left(x_{0}, r\right)\right)=0$ for every $r$. (Such a choice of the sequence is possible due to Step 2).

By Step 1, for every $r$ there exists a $q$-equiintegrable sequence $\left\{v_{n, r}\right\}$ such that

$$
\begin{aligned}
& v_{n, r} \rightarrow v \quad \text { weakly in } L^{q}\left(Q\left(x_{0}, r\right) ; \mathbb{R}^{d}\right), \\
& \mathscr{A} v_{n, r} \rightarrow 0 \quad \text { strongly in } W^{-1, s}\left(Q\left(x_{0}, r\right) ; \mathbb{R}^{l}\right) \text { for every } 1<s<q
\end{aligned}
$$

as $n \rightarrow+\infty$, and

$$
\lim _{n \rightarrow+\infty} \int_{Q\left(x_{0}, r\right)} g\left(x, v_{n, r}(x)\right) d x \leq \mathcal{I}\left((u, v), Q\left(x_{0}, r\right)\right)+r^{N+1} .
$$

A change of variables yields

$$
\frac{d \mathcal{I}((u, v), \cdot)}{d \mathcal{L}^{N}}\left(x_{0}\right) \geq \liminf _{r \rightarrow 0^{+}} \lim _{n \rightarrow+\infty} \int_{Q} g\left(x_{0}+r y, v\left(x_{0}\right)+w_{n, r}(y)\right) d y,
$$

where

$$
w_{n, r}(y):=v_{n, r}\left(x_{0}+r y\right)-v\left(x_{0}\right) \text { for a.e. } y \in Q .
$$

Arguing as in [6, Proof of Lemma 3.5], Hölder's inequality and a change of variables imply

$$
w_{n, r} \rightarrow 0 \quad \text { weakly in } L^{q}\left(Q ; \mathbb{R}^{d}\right)
$$

as $n \rightarrow+\infty$ and $r \rightarrow 0^{+}$, in this order. We claim that

$$
\mathscr{A}\left(x_{0}+r \cdot\right) w_{n, r} \rightarrow 0 \quad \text { strongly in } W^{-1, s}\left(Q ; \mathbb{R}^{l}\right),
$$

as $n \rightarrow+\infty$, for every $r$ and every $1<s<q$. 
Indeed, let $\varphi \in W_{0}^{1, s^{\prime}}\left(Q ; \mathbb{R}^{d}\right)$. There holds

$$
\begin{aligned}
\langle\mathscr{A} & \left.\left(x_{0}+r \cdot\right) w_{n, r}, \varphi\right\rangle_{W^{-1, s}\left(Q ; \mathbb{R}^{l}\right), W_{0}^{1, s^{\prime}}\left(Q ; \mathbb{R}^{l}\right)}=-\sum_{i=1}^{N}\left\{r \int_{Q} \frac{\partial A^{i}\left(x_{0}+r y\right)}{\partial x_{i}} v_{n, r}\left(x_{0}+r y\right) \cdot \varphi(y) d y\right. \\
& \left.+\int_{Q} A^{i}\left(x_{0}+r y\right) v_{n, r}\left(x_{0}+r y\right) \cdot \frac{\partial \varphi(y)}{\partial y_{i}} d y\right\} \\
= & -\sum_{i=1}^{N}\left\{\frac{1}{r^{N-1}} \int_{Q\left(x_{0}, r\right)} \frac{\partial A^{i}(x)}{\partial x_{i}} v_{n, r}(x) \cdot \psi_{r}(x) d x+\frac{1}{r^{N-1}} \int_{Q\left(x_{0}, r\right)} A^{i}(x) v_{n, r}(x) \cdot \frac{\partial \psi_{r}(x)}{\partial x_{i}} d x\right\} \\
= & \frac{1}{r^{N-1}}\left\langle\mathscr{A} v_{n, r}, \psi_{r}\right\rangle_{W^{-1, s}\left(Q\left(x_{0}, r\right) ; \mathbb{R}^{l}\right), W_{0}^{1, s^{\prime}}}\left(Q\left(x_{0}, r\right) ; \mathbb{R}^{l}\right),
\end{aligned}
$$

where $\psi_{r}(x):=\varphi\left(\frac{x-x_{0}}{r}\right)$ for a.e. $x \in Q\left(x_{0}, r\right)$. Since $\psi_{r} \in W_{0}^{1, s^{\prime}}\left(Q\left(x_{0}, r\right) ; \mathbb{R}^{d}\right)$ and

$$
\left\|\psi_{r}\right\|_{W_{0}^{1, s^{\prime}}\left(Q\left(x_{0}, r\right) ; \mathbb{R}^{d}\right)} \leq C(r)\|\varphi\|_{W_{0}^{1, s^{\prime}}\left(Q ; \mathbb{R}^{d}\right)},
$$

we obtain the estimate

$$
\left\|\mathscr{A}\left(x_{0}+r \cdot\right) w_{n, r}\right\|_{W^{-1, s}\left(Q ; \mathbb{R}^{l}\right)} \leq C(r)\left\|\mathscr{A} v_{n, r}\right\|_{W^{-1, s}\left(Q\left(x_{0}, r\right) ; \mathbb{R}^{l}\right)} .
$$

Claim (3.18) follows by (3.16).

In view of (3.17) and (3.18), a diagonalization procedure yields a $q$-equiintegrable sequence $\left\{\hat{w}_{k}\right\} \subset$ $L^{q}\left(Q ; \mathbb{R}^{d}\right)$ satisfying

$$
\begin{aligned}
& \hat{w}_{k} \rightarrow 0 \quad \text { weakly in } L^{q}\left(Q ; \mathbb{R}^{d}\right), \\
& \mathscr{A}\left(x_{0}+r_{k} \cdot\right) \hat{w}_{k} \rightarrow 0 \quad \text { strongly in } W^{-1, s}\left(Q ; \mathbb{R}^{l}\right) \text { for every } 1<s<q,
\end{aligned}
$$

and

$$
\frac{d \mathcal{I}((u, v), \cdot)}{d \mathcal{L}^{N}}\left(x_{0}\right) \geq \liminf _{k \rightarrow+\infty} \int_{Q} g\left(x_{0}+r_{k} y, v\left(x_{0}\right)+\hat{w}_{k}(y)\right) d y
$$

For every $\varphi \in W_{0}^{1, s^{\prime}}\left(Q ; \mathbb{R}^{l}\right), 1<s<q$, there holds

$$
\begin{aligned}
& \left\langle\left(\mathscr{A}\left(x_{0}+r_{k} \cdot\right)-\mathscr{A}\left(x_{0}\right)\right) \hat{w}_{k}, \varphi\right\rangle_{W^{-1, s}\left(Q ; \mathbb{R}^{l}\right), W_{0}^{1, s^{\prime}}\left(Q ; \mathbb{R}^{l}\right)} \\
& \quad=-\sum_{i=1}^{N}\left[r_{k} \int_{Q} \frac{\partial A^{i}\left(x_{0}+r_{k} y\right)}{\partial x_{i}} \hat{w}_{k}(y) \cdot \varphi(y) d y+\int_{Q}\left(A^{i}\left(x_{0}+r_{k} y\right)-A^{i}\left(x_{0}\right)\right) \hat{w}_{k}(y) \cdot \frac{\partial \varphi(y)}{\partial y_{i}} d y\right] .
\end{aligned}
$$

Thus,

$$
\left\|\left(\mathscr{A}\left(x_{0}+r_{k} \cdot\right)-\mathscr{A}\left(x_{0}\right)\right) \hat{w}_{k}\right\|_{W^{-1, s}\left(Q ; \mathbb{R}^{l}\right)} \leq r_{k} \sum_{i=1}^{N}\left\|A^{i}\right\|_{W^{1, \infty}\left(\mathbb{R}^{N} ; \mathbb{R}^{l \times d}\right)}\left\|\hat{w}_{k}\right\|_{L^{q}\left(Q ; \mathbb{R}^{d}\right)}
$$

for every $1<s<q$. By (3.19) and (3.20) we conclude that

$$
\mathscr{A}\left(x_{0}\right) \hat{w}_{k} \rightarrow 0 \quad \text { strongly in } W^{-1, s}\left(Q ; \mathbb{R}^{l}\right) \quad \text { for every } 1<s<q .
$$

In view of (3.19) and (3.22), an adaptation of [6, Corollary 3.3] yields a $q$-equiintegrable sequence $\left\{w_{k}\right\}$ such that

$$
\begin{aligned}
& w_{k} \rightarrow 0 \quad \text { weakly in } L^{q}\left(Q ; \mathbb{R}^{d}\right), \\
& \int_{Q} w_{k}(y) d y=0 \text { for every } k, \\
& \mathscr{A}\left(x_{0}\right) w_{k}=0 \text { for every } k,
\end{aligned}
$$

and

$$
\liminf _{k \rightarrow+\infty} \int_{Q} g\left(x_{0}, v\left(x_{0}\right)+w_{k}(y)\right) d y \leq \liminf _{k \rightarrow+\infty} \int_{Q} g\left(x_{0}+r_{k} y, v\left(x_{0}\right)+\hat{w}_{k}(y)\right) d y
$$


Finally, by combining (3.21), (3.23), and (3.24), and by the definition of $\mathscr{A}$-quasiconvex envelope for operators with constant coefficients, we obtain

$$
\begin{aligned}
\frac{d \mathcal{I}((u, v), \cdot)}{d \mathcal{L}^{N}}\left(x_{0}\right) & \geq \liminf _{k \rightarrow+\infty} \int_{Q} g\left(x_{0}, v\left(x_{0}\right)+w_{k}(y)\right) d y \\
& =\liminf _{k \rightarrow+\infty} \int_{Q} f\left(x_{0}, u\left(x_{0}\right), v\left(x_{0}\right)+w_{k}(y)\right) d y \geq Q_{\mathscr{A}\left(x_{0}\right)} f\left(x_{0}, u\left(x_{0}\right), v\left(x_{0}\right)\right)
\end{aligned}
$$

for a.e. $x_{0} \in \Omega$. This concludes the proof of Claim (3.12).

Step 4:

To complete the proof of the theorem we need to show that

$$
\frac{d \mathcal{I}((u, v), \cdot)}{d \mathcal{L}^{N}}\left(x_{0}\right) \leq Q_{\mathscr{A}\left(x_{0}\right)} f\left(x_{0}, u\left(x_{0}\right), v\left(x_{0}\right)\right) \quad \text { for a.e. } x_{0} \in \Omega .
$$

To this aim, let $\mu>0$, and $x_{0} \in \omega$ be such that (3.14) and (3.15) hold. Let $w \in C_{\mathrm{per}}^{\infty}\left(\mathbb{R}^{N} ; \mathbb{R}^{d}\right)$ be such that

$$
\int_{Q} w(y) d y=0, \quad \mathscr{A}\left(x_{0}\right) w=0
$$

and

$$
\int_{Q} f\left(x_{0}, u\left(x_{0}\right), v\left(x_{0}\right)+w(y)\right) d y \leq Q_{\mathscr{A}\left(x_{0}\right)} f\left(x_{0}, u\left(x_{0}\right), v\left(x_{0}\right)\right)+\mu .
$$

Let $\eta \in C_{c}^{\infty}(\Omega ;[0,1])$ be such that $\eta \equiv 1$ in a neighborhood of $x_{0}$ and let $r$ be small enough so that

$$
Q\left(x_{0}, r\right) \subset\{x: \eta(x)=1\} \quad \text { and } Q\left(x_{0}, 2 r\right) \subset \subset \Omega .
$$

Consider a map $\varphi \in C_{c}^{\infty}\left(Q\left(x_{0}, r\right) ;[0,1]\right)$ satisfying

$$
\mathcal{L}^{N}\left(Q\left(x_{0}, r\right) \cap\{\varphi \neq 1\}\right)<\mu r^{N},
$$

and define

$$
z_{m}^{r}(x):=\varphi(x) w\left(\frac{m\left(x-x_{0}\right)}{r}\right) \quad \text { for } x \in \mathbb{R}^{N} .
$$

We observe that $z_{m}^{r} \in L^{q}\left(\Omega ; \mathbb{R}^{d}\right)$, and for $\psi \in L^{q^{\prime}}\left(\Omega ; \mathbb{R}^{d}\right)$ we have

$$
\begin{aligned}
\int_{\Omega} z_{m}^{r}(x) \cdot \psi(x) d x & =\int_{\Omega} \varphi(x) w\left(\frac{m\left(x-x_{0}\right)}{r}\right) \cdot \psi(x) d x \\
& =r^{N} \int_{Q} \varphi\left(x_{0}+r y\right) w(m y) \cdot \psi\left(x_{0}+r y\right) d y .
\end{aligned}
$$

By (3.26) and by the Riemann-Lebesgue lemma we have

$$
z_{m}^{r} \rightarrow 0 \quad \text { weakly in } L^{q}\left(\Omega ; \mathbb{R}^{d}\right)
$$

as $m \rightarrow+\infty$. We claim that

$$
\limsup _{m \rightarrow+\infty}\left\|\mathscr{A}_{\eta} z_{m}^{r}\right\|_{W^{-1, q}\left(\Omega ; \mathbb{R}^{l}\right)} \leq C r^{\frac{N}{q}+1},
$$

where $\mathscr{A}_{\eta}$ is the pseudo-differential operator defined in (2.2). Indeed, by (3.28) we obtain

$$
\begin{aligned}
\mathscr{A}_{\eta} z_{m}^{r} & =\mathscr{A} z_{m}^{r}-\mathscr{A}\left(x_{0}\right) z_{m}^{r}+\mathscr{A}\left(x_{0}\right) z_{m}^{r} \\
& =\sum_{i=1}^{N} \frac{\partial\left(\left(A^{i}(x)-A^{i}\left(x_{0}\right)\right) z_{m}^{r}(x)\right)}{\partial x_{i}}+\sum_{i=1}^{N} A^{i}\left(x_{0}\right) \frac{\partial z_{m}^{r}(x)}{\partial x_{i}}-\sum_{i=1}^{N} \frac{\partial A^{i}(x)}{\partial x_{i}} z_{m}^{r}(x) .
\end{aligned}
$$


By the regularity of the operators $A^{i}$ and by a change of variables, the first term in the right-hand side of (3.33) is estimated as

$$
\begin{aligned}
& \left\|\sum_{i=1}^{N} \frac{\partial\left(\left(A^{i}(x)-A^{i}\left(x_{0}\right)\right) z_{m}^{r}(x)\right)}{\partial x_{i}}\right\|_{W^{-1, q}\left(\Omega ; \mathbb{R}^{l}\right)} \\
& \leq \sum_{i=1}^{N}\left\|\left(A^{i}(x)-A^{i}\left(x_{0}\right)\right) \varphi(x) w\left(\frac{m\left(x-x_{0}\right)}{r}\right)\right\|_{L^{q}\left(Q\left(x_{0}, r\right) ; \mathbb{R}^{l}\right)} \\
& \leq \sum_{i=1}^{N}\left\|A^{i}\right\|_{W^{1, \infty}\left(\mathbb{R}^{N} ; \mathbb{R}^{l \times d}\right)}\|\varphi\|_{L^{\infty}\left(Q\left(x_{0}, r\right)\right)}\|w(m \cdot)\|_{L^{q}\left(Q ; \mathbb{R}^{d}\right)} r^{\frac{N}{q}+1} \leq C r^{\frac{N}{q}+1} .
\end{aligned}
$$

In view of (3.26) the second term in the right-hand side of (3.33) becomes

$$
\sum_{i=1}^{N} A^{i}\left(x_{0}\right) \frac{\partial z_{m}^{r}(x)}{\partial x_{i}}=\sum_{i=1}^{N} A^{i}\left(x_{0}\right) \frac{\partial \varphi(x)}{\partial x_{i}} w\left(\frac{m\left(x-x_{0}\right)}{r}\right),
$$

and thus converges to zero weakly in $L^{q}\left(\Omega ; \mathbb{R}^{l}\right)$, as $m \rightarrow+\infty$, due to (3.26) and by the RiemannLebesgue lemma. Hence,

$$
\left\|\sum_{i=1}^{N} A^{i}\left(x_{0}\right) \frac{\partial z_{m}^{r}(x)}{\partial x_{i}}\right\|_{W^{-1, q}\left(\Omega ; \mathbb{R}^{l}\right)} \rightarrow 0 \quad \text { as } m \rightarrow+\infty
$$

by the compact embedding of $L^{q}\left(\Omega ; \mathbb{R}^{l}\right)$ into $W^{-1, q}\left(\Omega ; \mathbb{R}^{l}\right)$. Finally, the third term in the right-hand side of (3.33) satisfies

$$
\sum_{i=1}^{N} \frac{\partial A^{i}(x)}{\partial x_{i}} z_{m}^{r}(x)=\sum_{i=1}^{N} \frac{\partial A^{i}(x)}{\partial x_{i}} \varphi(x) w\left(\frac{m\left(x-x_{0}\right)}{r}\right),
$$

which again converges to zero weakly in $L^{q}\left(\Omega ; \mathbb{R}^{l}\right)$, as $m \rightarrow+\infty$, owing again to (3.26) and the Riemann-Lebesgue lemma. Therefore,

$$
\left\|\sum_{i=1}^{N} \frac{\partial A^{i}(x)}{\partial x_{i}} z_{m}^{r}(x)\right\|_{W^{-1, q}\left(\Omega ; \mathbb{R}^{l}\right)} \rightarrow 0 \quad \text { as } m \rightarrow+\infty .
$$

Claim (3.32) follows by combining (3.34)-(3.36).

Consider the maps

$$
v_{m}^{r}:=P_{\eta} z_{m}^{r}
$$

where $P_{\eta}$ is the projection operator introduced in (2.3). By Proposition 2.2 we have

$$
\begin{aligned}
& \left\|v_{m}^{r}\right\|_{L^{q}\left(Q\left(x_{0}, r\right) ; \mathbb{R}^{d}\right)} \leq C\left\|z_{m}^{r}\right\|_{L^{q}\left(\Omega ; \mathbb{R}^{d}\right)}, \\
& \left\|v_{m}^{r}\right\|_{W^{-1, q}\left(Q\left(x_{0}, r\right) ; \mathbb{R}^{d}\right)} \leq C\left\|z_{m}^{r}\right\|_{W^{-1, q}\left(\Omega ; \mathbb{R}^{d}\right)}, \\
& \left\|\mathscr{A}_{\eta} v_{m}^{r}\right\|_{W^{-1, q}\left(Q\left(x_{0}, r\right) ; \mathbb{R}^{l}\right)} \leq C\left\|z_{m}^{r}\right\|_{W^{-1, q}\left(\Omega ; \mathbb{R}^{d}\right)}, \\
& \left\|v_{m}^{r}-z_{m}^{r}\right\|_{L^{q}\left(Q\left(x_{0}, r\right) ; \mathbb{R}^{d}\right)} \leq C\left(\left\|\mathscr{A}_{\eta} z_{m}^{r}\right\|_{W^{-1, q}\left(\Omega ; \mathbb{R}^{l}\right)}+\left\|z_{m}^{r}\right\|_{W^{-1, q}\left(\Omega ; \mathbb{R}^{d}\right)}\right) .
\end{aligned}
$$

By (3.31) and (3.37), the sequence $\left\{v_{m}^{r}\right\}$ is uniformly bounded in $L^{q}\left(Q\left(x_{0}, r\right) ; \mathbb{R}^{d}\right)$. Thus, there exists a map $v^{r} \in L^{q}\left(Q\left(x_{0}, r\right) ; \mathbb{R}^{d}\right)$ such that, up to the extraction of a (not relabelled) subsequence,

$$
v_{m}^{r} \rightarrow v^{r} \quad \text { weakly in } L^{q}\left(Q\left(x_{0}, r\right) ; \mathbb{R}^{d}\right)
$$

as $m \rightarrow+\infty$. Again by (3.31), and by the compact embedding of $L^{q}$ into $W^{-1, q}$, we deduce that

$$
z_{m}^{r} \rightarrow 0 \quad \text { strongy in } W^{-1, q}\left(\Omega ; \mathbb{R}^{d}\right)
$$

as $m \rightarrow+\infty$. Therefore, by combining (3.38) and (3.41), we conclude that

$$
v_{m}^{r} \rightarrow 0 \quad \text { weakly in } L^{q}\left(Q\left(x_{0}, r\right) ; \mathbb{R}^{d}\right)
$$


as $m \rightarrow+\infty$, and the convergence holds for the entire sequence. Additionally, by (3.28), (3.39), and (3.42), we obtain

$$
\mathscr{A} v_{m}^{r}=\mathscr{A}_{\eta} v_{m}^{r} \rightarrow 0 \quad \text { strongly in } W^{-1, q}\left(Q\left(x_{0}, r\right) ; \mathbb{R}^{l}\right)
$$

as $m \rightarrow+\infty$. Finally, by (3.32), (3.40), and (3.42), there holds

$$
\lim _{r \rightarrow 0} \lim _{m \rightarrow+\infty} r^{-\frac{N}{q}}\left\|v_{m}^{r}-z_{m}^{r}\right\|_{L^{q}\left(Q\left(x_{0}, r\right) ; \mathbb{R}^{d}\right)}=0
$$

We recall that, since $x_{0}$ satisfies (3.15), Step 1 yields

$$
\frac{d \mathcal{I}(u, v)}{d \mathcal{L}^{N}}\left(x_{0}\right)=\lim _{r \rightarrow 0^{+}} \frac{\mathcal{I}\left((u, v) ; Q\left(x_{0}, r\right)\right)}{r^{N}} \leq \liminf _{r \rightarrow 0^{+}} \liminf _{m \rightarrow+\infty} \frac{1}{r^{N}} \int_{Q\left(x_{0}, r\right)} f\left(x, u(x), v(x)+v_{m}^{r}(x)\right) d x .
$$

We claim that

$$
\frac{d \mathcal{I}(u, v)}{d \mathcal{L}^{N}}\left(x_{0}\right)=\lim _{r \rightarrow 0^{+}} \frac{\mathcal{I}\left((u, v) ; Q\left(x_{0}, r\right)\right)}{r^{N}} \leq \liminf _{r \rightarrow 0^{+}} \liminf _{m \rightarrow+\infty} \frac{1}{r^{N}} \int_{Q\left(x_{0}, r\right)} g\left(x, v(x)+z_{m}^{r}(x)\right) d x,
$$

where $g$ is the function introduced in Step 3. Indeed, for every $r \in \mathbb{R}$, consider the function $g^{r}$ : $Q \times \mathbb{R}^{d} \rightarrow[0,+\infty)$ defined as

$$
g^{r}(y, \xi):=g\left(x_{0}+r y, \xi\right) \text { for every } y \in Q, \xi \in \mathbb{R}^{d} .
$$

Since $x_{0} \in \omega$, by (3.13) there exists $K_{j}$ such that $x_{0} \in K_{j}$. In particular, this yields the existence of $r_{0}>0$ such that for $r \leq r_{0}$, the maps $g^{r}$ are continuous on $Q \times \mathbb{R}^{d}$, and the family $\left\{g^{r}(y, \cdot)\right\}$ is equicontinuous in $\mathbb{R}^{d}$, uniformly with respect to $y$. A change of variables yields

$$
\begin{aligned}
& \frac{1}{r^{N}}\left|\int_{Q\left(x_{0}, r\right)} f\left(x, u(x), v(x)+v_{m}^{r}(x)\right) d x-\int_{Q\left(x_{0}, r\right)} f\left(x, u(x), v(x)+z_{m}^{r}(x)\right) d x\right| \\
& =\left|\int_{Q} g^{r}\left(y, v\left(x_{0}+r y\right)+v_{m}^{r}\left(x_{0}+r y\right)\right) d y-\int_{Q} g^{r}\left(y, v\left(x_{0}+r y\right)+z_{m}^{r}\left(x_{0}+r y\right)\right) d y\right| .
\end{aligned}
$$

On the other hand, by (3.43) we have

$$
\lim _{r \rightarrow 0} \lim _{m \rightarrow+\infty}\left\|z_{m}^{r}\left(x_{0}+r \cdot\right)-v_{m}^{r}\left(x_{0}+r \cdot\right)\right\|_{L^{q}\left(Q ; \mathbb{R}^{d}\right)}=\lim _{r \rightarrow 0} \lim _{m \rightarrow+\infty} r^{-\frac{N}{q}}\left\|z_{m}^{r}-v_{m}^{r}\right\|_{L^{q}\left(Q\left(x_{0}, r\right) ; \mathbb{R}^{d}\right)}=0 .
$$

Therefore, by a diagonal procedure we extract a subsequence $\left\{m_{r}\right\}$ such that

$$
\begin{aligned}
& \limsup _{r \rightarrow 0} \limsup _{m \rightarrow+\infty}\left|\int_{Q} g^{r}\left(y, v\left(x_{0}+r y\right)+v_{m}^{r}\left(x_{0}+r y\right)\right) d y-\int_{Q} g^{r}\left(y, v\left(x_{0}+r y\right)+z_{m}^{r}\left(x_{0}+r y\right)\right) d y\right| \\
& =\lim _{r \rightarrow 0}\left|\int_{Q} g^{r}\left(y, v\left(x_{0}+r y\right)+v_{m_{r}}^{r}\left(x_{0}+r y\right)\right) d y-\int_{Q} g^{r}\left(y, v\left(x_{0}+r y\right)+z_{m_{r}}^{r}\left(x_{0}+r y\right)\right) d y\right|,
\end{aligned}
$$

and

$$
z_{m_{r}}^{r}\left(x_{0}+r \cdot\right)-v_{m_{r}}^{r}\left(x_{0}+r \cdot\right) \rightarrow 0 \quad \text { strongly in } L^{q}\left(Q ; \mathbb{R}^{d}\right) .
$$

In view of $(3.14),(3.30)$ and the Riemann-Lebesgue lemma, the sequence $\left\{v\left(x_{0}+r \cdot\right)+z_{m_{r}}^{r}\left(x_{0}+r \cdot\right)\right\}$ is $q$-equiintegrable in $Q$. Hence, by $(\mathrm{H})$ we are under the assumptions of Proposition 3.2, and we conclude that

$$
\lim _{r \rightarrow 0}\left|\int_{Q} g^{r}\left(y, v\left(x_{0}+r y\right)+v_{m_{r}}^{r}\left(x_{0}+r y\right)\right) d y-\int_{Q} g^{r}\left(y, v\left(x_{0}+r y\right)+z_{m_{r}}^{r}\left(x_{0}+r y\right)\right) d y\right|=0 .
$$

Claim (3.45) follows by combining (3.46) with (3.47). 
Arguing as in [6, Proof of Lemma 3.5], for every $x_{0} \in \omega$ (where $\omega$ is the set defined in (3.13)) we have

$$
\begin{aligned}
& \liminf _{r \rightarrow 0^{+}} \liminf _{m \rightarrow+\infty} \frac{1}{r^{N}} \int_{Q\left(x_{0}, r\right)} f\left(x, u(x), v(x)+z_{m}^{r}(x)\right) d x \\
& \quad \leq \liminf _{r \rightarrow 0^{+}} \liminf _{m \rightarrow+\infty} \frac{1}{r^{N}} \int_{Q\left(x_{0}, r\right)} f\left(x_{0}, u\left(x_{0}\right), v\left(x_{0}\right)+z_{m}^{r}(x)\right) d x
\end{aligned}
$$

hence by (3.45) we deduce that

$$
\frac{d \mathcal{I}(u, v)}{d \mathcal{L}^{N}}\left(x_{0}\right) \leq \liminf _{r \rightarrow 0^{+}} \liminf _{m \rightarrow+\infty} \frac{1}{r^{N}} \int_{Q\left(x_{0}, r\right)} f\left(x_{0}, u\left(x_{0}\right), v\left(x_{0}\right)+z_{m}^{r}(x)\right) d x .
$$

By (3.30) we obtain

$$
\begin{aligned}
\frac{d \mathcal{I}(u, v)}{d \mathcal{L}^{N}}\left(x_{0}\right) \leq & \liminf _{r \rightarrow 0^{+}} \liminf _{m \rightarrow+\infty} \frac{1}{r^{N}} \int_{Q\left(x_{0}, r\right)} f\left(x_{0}, u\left(x_{0}\right), v\left(x_{0}\right)+z_{m}^{r}(x)\right) d x \\
\leq & \liminf _{r \rightarrow 0^{+}} \liminf _{m \rightarrow+\infty} \frac{1}{r^{N}}\left\{\int_{Q\left(x_{0}, r\right)} f\left(x_{0}, u\left(x_{0}\right), v\left(x_{0}\right)+w\left(\frac{m\left(x-x_{0}\right)}{r}\right)\right) d x\right. \\
& \left.+\int_{Q\left(x_{0}, r\right) \cap\{\varphi \neq 1\}} f\left(x_{0}, u\left(x_{0}\right), v\left(x_{0}\right)+\varphi(x) w\left(\frac{m\left(x-x_{0}\right)}{r}\right)\right) d x\right\} .
\end{aligned}
$$

The growth assumption (H) and estimate (3.29) yield

$$
\begin{aligned}
& \int_{Q\left(x_{0}, r\right) \cap\{\varphi \neq 1\}} f\left(x_{0}, u\left(x_{0}\right), v\left(x_{0}\right)+\varphi(x) w\left(\frac{m\left(x-x_{0}\right)}{r}\right)\right) d x \\
& \quad \leq C \int_{Q\left(x_{0}, r\right) \cap\{\varphi \neq 1\}}\left(1+\left|w\left(\frac{m\left(x-x_{0}\right)}{r}\right)\right|^{q}\right) d x \\
& \quad \leq C\left(1+\|w\|_{L^{\infty}\left(\mathbb{R}^{N} ; \mathbb{R}^{d}\right)}^{q}\right) \mathcal{L}^{N}\left(Q\left(x_{0}, r\right) \cap\{\varphi \neq 1\}\right) \leq C \mu r^{N} .
\end{aligned}
$$

Thus, by (3.48), the periodicity of $w$, and Riemann-Lebesgue lemma, we deduce

$$
\begin{aligned}
\frac{d \mathcal{I}(u, v)}{d \mathcal{L}^{N}}\left(x_{0}\right) & \leq C \mu+\liminf _{r \rightarrow 0^{+}} \liminf _{m \rightarrow+\infty} \frac{1}{r^{N}} \int_{Q\left(x_{0}, r\right)} f\left(x_{0}, u\left(x_{0}\right), v\left(x_{0}\right)+w\left(\frac{m\left(x-x_{0}\right)}{r}\right)\right) d x \\
& =C \mu+\liminf _{m \rightarrow+\infty} \int_{Q} f\left(x_{0}, u\left(x_{0}\right), v\left(x_{0}\right)+w(m y)\right) d y \\
& =C \mu+\int_{Q} f\left(x_{0}, u\left(x_{0}\right), v\left(x_{0}\right)+w(y)\right) d y \\
& \leq C \mu+Q_{\mathscr{A}\left(x_{0}\right)} f\left(x_{0}, u\left(x_{0}\right), v\left(x_{0}\right)\right)
\end{aligned}
$$

where the last inequality is due to (3.27). Letting $\mu \rightarrow 0^{+}$we conclude (3.25).

\section{ACKNOWLEDGEMENTS}

The authors thank the Center for Nonlinear Analysis (NSF Grant No. DMS-0635983), where this research was carried out, and also acknowledge support of the National Science Foundation under the PIRE Grant No. OISE-0967140. The research of I. Fonseca and E. Davoli was funded by the National Science Foundation under Grant No. DMS- 0905778. E. Davoli acknowledges the support of the Austrian Science Fund (FWF) projects P 27052 and I 2375. The research of I. Fonseca was further partially supported by the National Science Foundation under Grant No. DMS-1411646. 


\section{REFERENCES}

[1] Adolfo Arroyo-Rabasa. Relaxation and optimization for linear-growth convex integral functionals under PDE constraints. arXiv:1603.01310, 2016.

[2] Adolfo Arroyo-Rabasa, Guido De Philippis, and Filip Rindler. Lower semicontinuity and relaxation of linear-growth integral functionals under pde constraints. arXiv:1701.02230, 2017.

[3] Hedy Attouch. Variational convergence for functions and operators. Applicable Mathematics Series. Pitman (Advanced Publishing Program), Boston, MA, 1984.

[4] Margarida Baía, Milena Chermisi, José Matias, and Pedro M. Santos. Lower semicontinuity and relaxation of signed functionals with linear growth in the context of $\mathscr{A}$-quasiconvexity. Calc. Var. Partial Differential Equations, 47(3-4):465-498, 2013.

[5] Barbora Benešová and Martin Kružík. Weak lower semicontinuity of integral functionals and applications. arXiv:1601.00390v4, 2016.

[6] Andrea Braides, Irene Fonseca, and Giovanni Leoni. $\mathscr{A}$-quasiconvexity: relaxation and homogenization. ESAIM Control Optim. Calc. Var., 5:539-577 (electronic), 2000.

[7] Elisabetta Chiodaroli, Eduard Feireisl, Ondřej Kreml, and Emil Wiedemann. $\mathscr{A}$-free rigidity and applications to the compressible Euler system. Annali di Matematica Pura ed Applicata (1923 -), pages 1-16, 2017.

[8] Bernard Dacorogna. Weak continuity and weak lower semicontinuity of nonlinear functionals, volume 922 of Lecture Notes in Mathematics. Springer-Verlag, Berlin-New York, 1982.

[9] Bernard Dacorogna and Irene Fonseca. A-B quasiconvexity and implicit partial differential equations. Calc. Var. Partial Differential Equations, 14(2):115-149, 2002.

[10] Elisa Davoli and Irene Fonseca. Homogenization of integral energies under periodically oscillating differential constraints. Calc. Var. Partial Differential Equations, 55(3):1-60, 2016.

[11] Elisa Davoli and Irene Fonseca. Periodic homogenization of integral energies under space-dependent differential constraints. Port. Math., 73(4):279-317, 2016.

[12] Guido De Philippis and Filip Rindler. On the structure of $\mathcal{A}$-free measures and applications. Ann. of Math. (2), 184(3):1017-1039, 2016.

[13] Irene Fonseca and Stefan Krömer. Multiple integrals under differential constraints: two-scale convergence and homogenization. Indiana Univ. Math. J., 59(2):427-457, 2010.

[14] Irene Fonseca and Martin Kružík. Oscillations and concentrations generated by $\mathscr{A}$-free mappings and weak lower semicontinuity of integral functionals. ESAIM Control Optim. Calc. Var., 16(2):472-502, 2010.

[15] Irene Fonseca, Giovanni Leoni, and Stefan Müller. A-quasiconvexity: weak-star convergence and the gap. Ann. Inst. H. Poincaré Anal. Non Linéaire, 21(2):209-236, 2004.

[16] Irene Fonseca and Stefan Müller. Relaxation of quasiconvex functionals in $\operatorname{BV}\left(\Omega, \mathbf{R}^{p}\right)$ for integrands $f(x, u, \nabla u)$. Arch. Rational Mech. Anal., 123(1):1-49, 1993.

[17] Irene Fonseca and Stefan Müller. $\mathscr{A}$-quasiconvexity, lower semicontinuity, and Young measures. SIAM J. Math. Anal., 30(6):1355-1390 (electronic), 1999.

[18] Jan Krämer, Stefan Krömer, Martin Kružík, and Gabriel Pathó. $\mathscr{A}$-quasiconvexity at the boundary and weak lower semicontinuity of integral functionals. Adv. Calc. Var., 10(1):49-67, 2017.

[19] Carolin Kreisbeck and Stefan Krömer. Heterogeneous thin films: combining homogenization and dimension reduction with directors. SIAM J. Math. Anal., 48(2):785-820, 2016.

[20] Carolin Kreisbeck and Filip Rindler. Thin-film limits of functionals on $\mathscr{A}$-free vector fields. Indiana Univ. Math. J., 64(5):1383-1423, 2015.

[21] José Matias, Marco Morandotti, and Pedro M. Santos. Homogenization of functionals with linear growth in the context of $\mathcal{A}$-quasiconvexity. Appl. Math. Optim., 72(3):523-547, 2015.

[22] François Murat. Compacité par compensation: condition nécessaire et suffisante de continuité faible sous une hypothèse de rang constant. Ann. Scuola Norm. Sup. Pisa Cl. Sci. (4), 8(1):69-102, 1981.

[23] Pedro M. Santos. ÁA-quasi-convexity with variable coefficients. Proc. Roy. Soc. Edinburgh Sect. A, 134(6):1219-1237, 2004.

(Elisa Davoli) Faculty of Mathematics, University of Vienna, Oskar-Morgenstern Platz 1, A-1090 Vienna, AUSTRIA

E-mail address, E. Davoli: elisa.davoli@univie.ac.at

(Irene Fonseca) Department of Mathematics, Carnegie Mellon University, Forbes Avenue, Pittsburgh PA 15213, USA

E-mail address, I. Fonseca: fonseca@andrew.cmu.edu 